\title{
The ergodic shadowing property from the robust and generic view point
}

Manseob Lee*

"Correspondence: Imsds@mokwon.ac.kr

Department of Mathematics, Mokwon University, Daejeon, 302-729, Korea

\begin{abstract}
In this paper, we discuss that if a diffeomorphisms has the $C^{1}$-stably ergodic shadowing property in a closed set, then it is a hyperbolic elementary set. Moreover, $C^{1}$-generically: if a diffeomorphism has the ergodic shadowing property in a locally maximal closed set, then it is a hyperbolic basic set.
\end{abstract}

MSC: 34D30; 37C20

Keywords: ergodic shadowing; shadowing; locally maximal; generic; Anosov

\section{Introduction}

Let $M$ be a closed $C^{\infty}$ manifold, and let $\operatorname{Diff}(M)$ be the space of diffeomorphisms of $M$ endowed with the $C^{1}$-topology. Denote by $d$ the distance on $M$ induced from a Riemannian metric $\|\cdot\|$ on the tangent bundle $T M$. Let $f \in \operatorname{Diff}(M)$. For $\delta>0$, a sequence of points $\left\{x_{i}\right\}_{i=a}^{b}(-\infty \leq a<b \leq \infty)$ in $M$ is called a $\delta$-pseudo orbit of $f$ if $d\left(f\left(x_{i}\right), x_{i+1}\right)<\delta$ for all $a \leq i \leq b-1$. For given $x, y \in M$, we write $x \rightsquigarrow y$ if for any $\delta>0$, there is a $\delta$-pseudo orbit $\left\{x_{i}\right\}_{i=a}^{b}(a<b)$ of $f$ such that $x_{a}=x$ and $x_{b}=y$. Let $\Lambda$ be a closed $f$-invariant set. We say that $f$ has the shadowing property in $\Lambda$ if for every $\epsilon>0$ there is $\delta>0$ such that, for any $\delta$-pseudo orbit $\left\{x_{i}\right\}_{i=a}^{b} \subset \Lambda$ of $f(-\infty \leq a<b \leq \infty)$, there is a point $y \in \Lambda$ such that $d\left(f^{i}(y), x_{i}\right)<\epsilon$ for all $a \leq i \leq b-1$. If $\Lambda=M$, then $f$ has the shadowing property. The shadowing property usually plays an important role in the investigation of stability theory and ergodic theory. For instance, Sakai [1] proved that if $f$ has the $C^{1}$-robustly shadowing property, then $f$ is structurally stable. Now we introduce the notion of the ergodic shadowing property which was introduced and studied by [2]. Lee has shown in [3] that if $f$ belongs to the $C^{1}$-interior of the set of all diffeomorphisms having the ergodic shadowing property, then it is structurally stable diffeomorphisms. In [4], Lee showed that if $f$ is local star condition and has the ergodic shadowing property on the homoclinic class, then it is hyperbolic. For any $\delta>0$, a sequence $\xi=\left\{x_{i}\right\}_{i \in \mathbb{Z}}$ is a $\delta$-ergodic pseudo orbit of $f$ if for $N p_{n}^{+}(\xi, f, \delta)=\left\{i: d\left(f\left(x_{i}\right), x_{i+1}\right) \geq \delta\right\} \cap\{0,1, \ldots, n-1\}$, and $N p_{n}^{-}(\xi, f, \delta)=\left\{-i: d\left(f^{-1}\left(x_{-i}\right), x_{-i-1}\right) \geq \delta\right\} \cap\{-n+1, \ldots,-1,0\}$

$$
\lim _{n \rightarrow \infty} \frac{\# N p_{n}^{+}(\xi, f, \delta)}{n}=0 \text { and } \lim _{n \rightarrow-\infty} \frac{\# N p_{n}^{-}(\xi, f, \delta)}{n}=0 .
$$

Here \#A is the number of elements of the set $A$. We say that $f$ has the ergodic shadowing property in $\Lambda$ (or $\left.f\right|_{\Lambda}$ has ergodic shadowing) if for any $\epsilon>0$, there is a $\delta>0$ such that every $\delta$-ergodic pseudo orbit $\xi=\left\{x_{i}\right\}_{i \in \mathbb{Z}} \subset \Lambda$ of $f$ there is a point $z \in \Lambda$ such that, for

(c) 2014 Lee; licensee Springer. This is an Open Access article distributed under the terms of the Creative Commons Attribution License (http://creativecommons.org/licenses/by/2.0), which permits unrestricted use, distribution, and reproduction in any medium, provided the original work is properly cited. 
$N s_{n}^{+}(\xi, f, z, \epsilon)=\left\{i: d\left(f^{i}(z), x_{i}\right) \geq \epsilon\right\} \cap\{0,1, \ldots, n-1\}$, and $N s_{n}^{-}(\xi, f, z, \epsilon)=\left\{-i: d\left(f^{-i}(z), x_{-i}\right) \geq\right.$

$\epsilon\} \cap\{-n+1, \ldots,-1,0\}$,

$$
\lim _{n \rightarrow \infty} \frac{\# N s_{n}^{+}(\xi, f, z, \epsilon)}{n}=0 \text { and } \lim _{n \rightarrow-\infty} \frac{\# N s_{n}^{-}(\xi, f, z, \epsilon)}{n}=0
$$

Note that $f$ has the ergodic shadowing property on $\Lambda$ and $f$ has the ergodic shadowing property in $\Lambda$ are different notions. That is, the shadowing point is in $M$ or $\Lambda$. In the first notion, the shadowing point is in $M$. In the second notion, the shadowing point is in $\Lambda$. In this paper we consider the latter case.

We say that $\Lambda$ is locally maximal if there is a compact neighborhood $U$ of $\Lambda$ such that

$$
\bigcap_{n \in \mathbb{Z}} f^{n}(U)=\Lambda_{f}(U)=\Lambda
$$

Now, we introduce the notion of the $C^{1}$-stably ergodic shadowing property in a closed set.

Definition 1.1 Let $\Lambda$ be a closed $f$-invariant set. We say that $f$ has the $C^{1}$-stably ergodic shadowing property in $\Lambda$ if

(i) there is a neighborhood $U$ of $\Lambda$ and a $C^{1}$-neighborhood $\mathcal{U}(f)$ of $f$ such that $\Lambda_{f}(U)=\Lambda=\bigcap_{n \in \mathbb{Z}} f^{n}(U)$ (that is, $\Lambda$ is locally maximal);

(ii) for any $g \in \mathcal{U}(f), g$ has the ergodic shadowing property on $\Lambda_{g}(U)=\bigcap_{n \in \mathbb{Z}} g^{n}(U)$, where $\Lambda_{g}(U)$ is the continuation of $\Lambda$.

We say that $\Lambda$ is hyperbolic if the tangent bundle $T_{\Lambda} M$ has a $D f$-invariant splitting $E^{s} \oplus$ $E^{u}$ and there exist constants $C>0$ and $0<\lambda<1$ such that

$$
\left\|\left.D_{x} f^{n}\right|_{E_{x}^{s}}\right\| \leq C \lambda^{n} \quad \text { and } \quad\left\|\left.D_{x} f^{-n}\right|_{E_{x}^{u}}\right\| \leq C \lambda^{n}
$$

for all $x \in \Lambda$ and $n \geq 0$. If $\Lambda=M$, then $f$ is Anosov. We say that $\Lambda$ is a basic set (resp. elementary set) if $\left.f\right|_{\Lambda}$ is transitive (resp. mixing) and locally maximal. Note that if $\Lambda$ is hyperbolic, then we can easily show that there is a periodic point such that the orbit of the periodic point is dense in the set. Then we get the following.

Theorem 1.2 [5, Theorem 3.3] Let $\Lambda$ be a closed f-invariant set. If f has the $C^{1}$-stably ergodic shadowing property in $\Lambda$, then it is a hyperbolic elementary set.

Corollary 1.3 If $f$ belongs to the $C^{1}$-interior of the set of all diffeomorphisms having the ergodic shadowing property, then it is transitive Anosov.

We say that a subset $\mathcal{G} \subset \operatorname{Diff}(M)$ is residual if $\mathcal{G}$ contains the intersection of a countable family of open and dense subsets of $\operatorname{Diff}(M)$; in this case $\mathcal{G}$ is dense in $\operatorname{Diff}(M)$. A property $\mathrm{P}$ is said to be $C^{1}$-generic if $\mathrm{P}$ holds for all diffeomorphisms which belong to some residual subset of $\operatorname{Diff}(M)$. We use the terminology 'for $C^{1}$-generic $f$ ' to express 'there is a residual subset $\mathcal{G} \subset \operatorname{Diff}(M)$ such that, for any $f \in \mathcal{G} \ldots$. In [6], Abdenur and Díaz proved that if tame diffeomorphisms has the shadowing property, then it is hyperbolic. Still open is the question if $C^{1}$-generically: $f$ is shadowable, then is it hyperbolic? 
Recently, Ahn et al. [7] have given a partial answer which is $C^{1}$-generically: if a locally maximal homoclinic class is shadowing, then it is hyperbolic. Lee has shown in [8] that $C^{1}$-generically: if $f$ has the limit shadowing property on the homoclinic class, then it is hyperbolic. Inspired by this, we consider that $C^{1}$-generically: $f$ has the ergodic shadowing property in a locally maximal closed set. Then we have the following.

Theorem 1.4 For $C^{1}$-generic $f$, iff has the ergodic shadowing property in a locally maximal closed set $\Lambda$, then it is a hyperbolic elementary set. Moreover, $C^{1}$-generically: iff has the ergodic shadowing property, then it is transitive Anosov.

\section{Proof of Theorem 1.4}

Let $P(f)$ be the set of periodic points of $f$. If $\left.f\right|_{\Lambda}$ is transitive, then every $p \in \Lambda \cap P(f)$ is saddle, that is, there is no eigenvalues of $D_{p} f^{\pi(p)}$ with modulus equal to 1 , at least one of them is greater than 1 , at least one of them is smaller than 1 , where $\pi(p)$ is the minimum period of $p$.

Lemma 2.1 [2, Corollary 3.5] If $f$ has the ergodic shadowing property in $\Lambda$, then $\left.f\right|_{\Lambda}$ is mixing.

By Lemma 2.1, $f$ has the ergodic shadowing property in $\Lambda$, then $\left.f\right|_{\Lambda}$ is mixing, and so $\left.f\right|_{\Lambda}$ is transitive. Thus $p \in \Lambda \cap P(f)$ is neither a sink nor a source.

Lemma 2.2 [2, Lemma 3.2] If $f$ has the ergodic shadowing property in $\Lambda$, then $f$ has a finite shadowing property in $\Lambda$.

We say that $f$ has the finite shadowing property on $\Lambda$ if for any $\epsilon>0$ there is $\delta>0$ such that, for any finite $\delta$-pseudo orbit $\left\{x_{0}, x_{1}, \ldots, x_{n}\right\} \subset \Lambda$, there is $y \in M$ such that $d\left(f^{i}(y), x_{i}\right)<\epsilon$ for all $0 \leq i<n$. In [9, Lemma 1.1.1], Pilyugin showed that $f$ has a finite shadowing shadowing property on $\Lambda$, then $f$ has the shadowing property on $\Lambda$.

Lemma 2.3 Let $f$ have the ergodic shadowing in $\Lambda$ and $\Lambda$ be locally maximal in $U$. Then the shadowing point taken from $\Lambda$.

Proof Let $f$ have the ergodic shadowing property in $\Lambda$, and let $U$ be a locally maximal of $\Lambda$. For any $\epsilon>0$, let $\delta>0$ be the number of the ergodic shadowing property of $f$. Take a sequence $\gamma=\left\{x_{i}\right\}_{i=0}^{n}(n \geq 1)$ such that $\gamma$ is a $\delta$-pseudo orbit of $f$ and $\gamma \subset \Lambda$. As in the proof of [2, Lemma 3.1], there is a $\delta$-pseudo orbit $\eta=\left\{x_{i}\right\}_{i=n}^{0}$ such that $\eta \subset \Lambda$. Then we set $\xi=\{\ldots, \gamma, \eta, \gamma, \eta, \ldots\}$ is a $\delta$-ergodic pseudo orbit of $f$. Clear that $\xi \subset \Lambda$. Since $f$ has the ergodic shadowing property in $\Lambda$, $\xi$ can be ergodic shadowed by some point $y \in \Lambda$. By Lemma 2.2, there is $\gamma \in \xi$ such that $d\left(f^{i}(y), x_{i}\right)<\epsilon$ for $0 \leq i \leq n-1$. By [9, Lemma 1.1.1], $f$ has the shadowing property on $\Lambda$. Since $\Lambda$ is locally maximal in $U$, the shadowing point $y \in \Lambda$.

Let $p \in P(f)$ be a hyperbolic saddle with period $\pi(p)>0$. Then there are the local stable manifold $W_{\epsilon}^{s}(p)$ and the local unstable manifold $W_{\epsilon}^{u}(p)$ of $p$ for some $\epsilon=\epsilon(p)>0$. It is easily seen that if $d\left(f^{n}(x), f^{n}(p)\right) \leq \epsilon$ for all $n \geq 0$, then $x \in W_{\epsilon}^{s}(p)$, and if $d\left(f^{n}(x), f^{n}(p)\right) \leq \epsilon$ for all $n \leq 0$, then $x \in W_{\epsilon}^{u}(p)$. The stable manifold $W^{s}(p)$ and the unstable manifold $W^{u}(p)$ 
defined as following. It is well known that if $p$ is a hyperbolic periodic point of $f$ with period $k$, then the sets

$$
\begin{aligned}
& W^{s}(p)=\left\{x \in M: f^{k n}(x) \rightarrow p \text { as } n \rightarrow \infty\right\} \text { and } \\
& W^{u}(p)=\left\{x \in M: f^{-k n}(x) \rightarrow p \text { as } n \rightarrow \infty\right\}
\end{aligned}
$$

are $C^{1}$-injectively immersed submanifolds of $M$.

Lemma 2.4 Let $p, q \in P(f)$ be hyperbolic saddles. Iff has the ergodic shadowing property in a closed set $\Lambda$, then $W^{s}(p) \cap W^{u}(q) \neq \emptyset$, and $W^{u}(p) \cap W^{s}(q) \neq \emptyset$.

Proof Let $p, q \in P(f)$ be hyperbolic saddles, and let $U$ be a locally maximal neighborhood of $\Lambda$. Suppose that $f$ has the ergodic shadowing property in a locally maximal $\Lambda$. Since $p$ and $q$ are hyperbolic, there are $\epsilon(p)>0$ and $\epsilon(q)>0$ as in the above. Take $\epsilon=\min \{\epsilon(p), \epsilon(q)\} / 4$ and let $0<\delta \leq \epsilon$ be the number of the ergodic shadowing property of $f$. For simplicity, we may assume that $f(p)=p$ and $f(q)=q$. Since $f$ has the ergodic shadowing property in $\Lambda,\left.f\right|_{\Lambda}$ is chain transitive. Then we can construct a finite $\delta$-pseudo orbit form $p$ to $q$ as follows: $x_{0}=p, x_{n}=q(n \geq 1)$, and $d\left(f\left(x_{i}\right), x_{i+1}\right)<\delta$ for all $0<i<n-1$. Put (i) $x_{-i}=f^{-i}(p)$, for all $i \leq 0$, and (ii) $x_{n+i}=f^{i}(q)$ for all $i \geq 0$. Then we have the sequence $\xi=\left\{x_{i}\right\}_{i \in \mathbb{Z}}=\left\{\ldots, p, x_{1}, x_{2}, \ldots, x_{n}, x_{n+1}, \ldots\right\}$. It is clearly a $\delta$-ergodic pseudo orbit of $f$. Since $f$ has the ergodic shadowing property in $\Lambda$ and locally maximal, by Lemma $2.2, f$ has the finite shadowing property on $\Lambda$ and so, by [9, Lemma 1.1.1], $f$ has the shadowing property in $\Lambda$. By the shadowing property in $\Lambda$, we can show that $\operatorname{Orb}(y) \subset W^{u}(p) \cap W^{s}(q)$, and so $W^{u}(p) \cap W^{s}(q) \neq \emptyset$. The other case is similar.

A diffeomorphism $f$ is Kupka-Smale if their periodic points of $f$ are hyperbolic and if $p, q \in P(f)$, then $W^{s}(p)$ is transversal to $W^{u}(q)$. Then it is $C^{1}$-residual in $\operatorname{Diff}(M)$. Denote by $\mathcal{K S}(M)$ the set of all Kupka-Smale diffeomorphisms. The following was proved by [10].

Lemma 2.5 [10, Lemma 2.4] Let $\Lambda$ be locally maximal in $U$, and let $\mathcal{U}(f)$ be given. If for any $g \in \mathcal{U}(f), p \in \Lambda_{g}(U) \cap P(g)$ is not hyperbolic, then there is $g_{1} \in \mathcal{U}(f)$ such that $g_{1}$ has two hyperbolic periodic points $p, q \in \Lambda_{g_{1}}(U)$ with different indices.

Denote by $\mathcal{F}(M)$ the set of $f \in \operatorname{Diff}(M)$ such that there is a $C^{1}$ neighborhood $\mathcal{U}(f)$ of $f$ such that, for any $g \in \mathcal{U}(f)$, every $p \in P(g)$ is hyperbolic. In [11], Hayashi proved that $f \in \mathcal{F}(M)$ if and only if $f$ satisfies both Axiom A and the no-cycle condition. We say that $f$ is the local star condition diffeomorphism if there exist a $C^{1}$-neighborhood $\mathcal{U}(f)$ and a neighborhood $U$ of $\Lambda$ such that, for any $g \in \mathcal{U}(f)$, every $p \in \Lambda_{g}(U) \cap P(g)$ is hyperbolic (see [12]). Denote by $\mathcal{F}(\Lambda)$ the set of all local star diffeomorphisms. Note that there are a $C^{1}$-neighborhood $\mathcal{U}(f)$ and a neighborhood $U$ of $p$ such that, for all $g \in \mathcal{U}(f)$, there is a unique hyperbolic periodic point $p_{g} \in U$ of $g$ with the same period as $p$ and $\operatorname{index}\left(p_{g}\right)=$ index $(p)$. Here index $(p)=\operatorname{dim} E_{p}^{s}$, and the point $p_{g}$ is called the continuation of $p$.

Lemma 2.6 [13, Lemma 2.2] There is a residual set $\mathcal{G}_{1} \subset \operatorname{Diff}(M)$ such that, for any $f \in \mathcal{G}_{1}$, iffor any $C^{1}$-neighborhood $\mathcal{U}(f)$ off, there exists $g \in \mathcal{U}(f)$ such that two hyperbolic periodic points $p_{g}, q_{g} \in P(g)$ with index $\left(p_{g}\right) \neq \operatorname{index}\left(q_{g}\right)$, then $f$ has two hyperbolic periodic points $p, q \in P(f)$ with index $(p) \neq \operatorname{index}(q)$. 
Lemma 2.7 There is a residual set $\mathcal{G}_{2} \subset \operatorname{Diff}(M)$ such that, for any $f \in \mathcal{G}_{2}$, iff has the ergodic shadowing property in a locally maximal $\Lambda$, then for any $p, q \in \Lambda \cap P(f)$

$$
\operatorname{index}(p)=\operatorname{index}(q) .
$$

Proof Let $f \in \mathcal{G}_{2}=\mathcal{G}_{1} \cap \mathcal{K} \mathcal{S}(M)$, and let $p, q \in \Lambda \cap P(f)$ be hyperbolic saddles. Suppose that $f$ has the ergodic shadowing property in a locally maximal $\Lambda$. Then by Lemma 2.4 $W^{s}(p) \cap W^{u}(q) \neq \emptyset$ and $W^{u}(p) \cap W^{s}(q) \neq \emptyset$. Since $f \in \mathcal{G}_{2}, W^{s}(p) \pitchfork W^{u}(q) \neq \emptyset$ and $W^{u}(p) \pitchfork$ $W^{s}(q) \neq \emptyset$. This means that $p \sim q$ and so index $(p)=\operatorname{index}(q)$.

Let $p$ be a periodic point of $f$. For $0<\delta<1$, we say that $p$ has a $\delta$-weak eigenvalue if $D f^{\pi(p)}(p)$ has an eigenvalue $\lambda$ such that $(1-\delta)^{\pi(p)}<|\lambda|<(1+\delta)^{\pi(p)}$. We say that a periodic point has a real spectrum if all of its eigenvalues are real and simple spectrum if all its eigenvalues have multiplicity one. Denote by $P_{h}(f)$ the set of all hyperbolic periodic points of $f$.

Lemma 2.8 [14, Lemma 5.1] There is a residual set $\mathcal{G}_{3} \subset \operatorname{Diff}(M)$ such that, for any $f \in \mathcal{G}_{3}$ :

- For any $\delta>0$, if for any $C^{1}$-neighborhood $\mathcal{U}(f)$ off there exist $g \in \mathcal{U}(f)$ and $p_{g} \in P_{h}(g)$ with a $\delta$-weak eigenvalue, then there is $p \in P_{h}(f)$ with a $2 \delta$-weak eigenvalue.

- For any $\delta>0$, if $q \in P_{h}(f)$ with a $\delta$-weak eigenvalue and a real spectrum, then there is $p \in P_{h}(f)$ with a $\delta$-weak eigenvalue with a simple real spectrum.

Lemma 2.9 There is a residual set $\mathcal{G}_{4} \subset \operatorname{Diff}(M)$ such that, for any $f \in \mathcal{G}_{4}$, if $f$ has the ergodic shadowing property in a locally maximal $\Lambda$, then there exists $\eta>0$ such that, for any $q \in \Lambda \cap P_{h}(f)$, $q$ has no $\eta$-weak eigenvalues.

Proof Let $f \in \mathcal{G}_{4}=\mathcal{G}_{2} \cap \mathcal{G}_{3}$ have the ergodic shadowing property in a locally maximal $\Lambda$. We will derive a contradiction. Suppose that, for any $\eta>0$, there is $q \in \Lambda \cap P_{h}(f)$ such that $q$ has an $\eta$-weak eigenvalue. By Franks' lemma, there is $g C^{1}$-close to $f$ such that $p$ is not hyperbolic. By Franks' lemma and Lemma 2.5, there is $h C^{1}$-nearby $g$ and $C^{1}$-close to $f$ such that $h$ has tow hyperbolic periodic points $q_{h}, \gamma_{h}$ with different indices. Since $f \in \mathcal{G}_{1}$, and it is locally maximal, by Lemma $2.6 f$ has two hyperbolic periodic points $q, \gamma$ in $\Lambda$. Since $f$ has the ergodic shadowing property in $\Lambda$, this is a contradiction by Lemma 2.7 .

Proposition 2.10 There is a residual set $\mathcal{G}_{4} \subset \operatorname{Diff}(M)$ such that, for any $f \in \mathcal{G}_{4}$, iff has the ergodic shadowing property in $\Lambda$, then $f \in \mathcal{F}(\Lambda)$.

Proof Let $f \in \mathcal{G}_{4}$ have the ergodic shadowing property in a locally maximal $\Lambda$. Suppose by contradiction that $f \notin \mathcal{F}(\Lambda)$. Then there are $g C^{1}$-close to $f$ and $q \in P(g)$ such that $q$ has a $\eta$-weak eigenvalue. Then by Lemma 2.9, we get a contradiction. Thus $f \in \mathcal{F}(\Lambda)$.

Proposition 2.11 [15, Proposition A] Let $f \in \mathcal{G}_{4}$, and let $\Lambda$ be locally maximal. Iff has the ergodic shadowing property in $\Lambda$, then there are $m>0, C \geq 1$ and $\lambda \in(0,1)$ such that, for any $p \in \Lambda \cap P(f)$ with $\pi(q)>m$, we have

$$
\prod_{i=0}^{\pi(p)-1}\left\|\left.D f^{m}\right|_{E^{s}\left(f^{m i}(x)\right)}\right\| \leq C \lambda^{\pi(p)},
$$




$$
\begin{aligned}
& \prod_{i=0}^{\pi(p)-1}\left\|\left.D f^{m}\right|_{E^{s}\left(f^{m i}(x)\right)}\right\| \leq C \lambda^{\pi(p)} \quad \text { and } \\
& \left\|\left.D f^{m}\right|_{E^{s}(x)}\right\| \cdot\left\|\left.D f^{-m}\right|_{E^{u}\left(f^{m}(x)\right)}\right\| \leq \lambda,
\end{aligned}
$$

where $\pi(p)$ is the period of $p$.

Remark 2.12 By Pugh's closing lemma, there is a residual set $\mathcal{G}_{6} \subset \operatorname{Diff}(M)$ such that, for any $f \in \mathcal{G}_{6}$, if $\left.f\right|_{\Lambda}$ is transitive, then there is a periodic orbit $p_{n}$ such that $\operatorname{Orb}\left(p_{n}\right) \rightarrow \Lambda$ in Hausdorff metric.

Lemma 2.13 [16, Theorem 3.8] There is residual set $\mathcal{G}_{5} \subset \operatorname{Diff}(M)$ such that, for any $f \in \mathcal{G}_{5}$, for any ergodic measure $\mu$ off, there is a sequence of the periodic point $p_{n}$ such that $\mu_{p_{n}} \rightarrow \mu$ in weak* topology and $\operatorname{Orb}\left(p_{n}\right) \rightarrow \operatorname{Supp}(\mu)$ in Hausdorff metric.

The following was proved by Mañé [17]. Denote by $\mathcal{M}\left(\left.f\right|_{\Lambda}\right)$ the set of invariant probabilities on the Borel $\sigma$-algebra of $\Lambda$ endowed with the weak* topology.

Lemma 2.14 Let $\Lambda \subset M$ be a closed $f$-invariant set off and $E \subset T_{\Lambda} M$ be a continuous invariant subbundle. If there is $m>0$ such that

$$
\int \log \left\|\left.D f^{m}\right|_{E}\right\| d \mu<0
$$

for every ergodic $\mu \in \mathcal{M}\left(\left.f^{m}\right|_{\Lambda}\right)$, then $E$ is contracting.

Proof of Theorem 1.4 Let $f \in \mathcal{G}_{4} \cap \mathcal{G}_{5} \cap \mathcal{G}_{6}$ have the ergodic shadowing property in a locally maximal $\Lambda$. Then by Proposition 2.11, we know that $\Lambda$ admits a dominated splitting $T_{\Lambda} M=E \oplus F$. Since $f$ has the ergodic shadowing property in $\Lambda$, by Lemma $2.1, \operatorname{Re}-$ mark 2.12, and Lemma 2.13, there is a sequence of periodic points such that $\operatorname{Orb}\left(p_{n}\right) \rightarrow$ $\operatorname{Supp}(\mu)=\Lambda$ in the Hausdorff metric. By Proposition 2.11, we have

$$
\int\left\|\left.D f^{m}\right|_{E}\right\| d \mu=\lim _{n \rightarrow \infty} \int\left\|\left.D f^{m}\right|_{E}\right\| d \mu_{p_{n}}<0 .
$$

By Lemma 2.14, $E$ is contracting. Similarly, we can show that $F$ is expanding.

Corollary 2.15 For $C^{1}$-generic $f$, iff has the ergodic shadowing property, then $f$ is transitive Anosov. 


\section{References}

1. Sakai, K: Pseudo orbit tracing property and strong transversality of diffeomorphisms on closed manifolds. Osaka J. Math. 31, 373-386 (1994)

2. Fakhari, A, Ghane, FH: On shadowing: ordinary and ergodic. J. Math. Anal. Appl. 364, 151-155 (2010)

3. Lee, M: Diffeomorphisms with robustly ergodic shadowing. Dyn. Contin. Discrete Impuls. Syst., Ser. A Math. Anal. 20 747-753 (2013)

4. Lee, M: The ergodic shadowing property and homoclinic classes. J. Inequal. Appl. 2014, 90 (2014)

5. Barzanouni, A, Honary, B: C $C^{1}$-Stable ergodic shadowable invariant sets and hyperbolicity. Gen. Math. Notes 9, 1-6 (2012)

6. Abdenur, F, Díaz, LJ: Pseudo-orbit shadowing in the $C^{1}$ topology. Discrete Contin. Dyn. Syst. 17, $223-245$ (2007)

7. Ahn, J, Lee, K, Lee, M: Homoclinic classes with shadowing. J. Inequal. Appl. 2012, 97 (2012)

8. Lee, M: Usual limit shadowable homoclinic classes of generic diffeomorphisms. Adv. Differ. Equ. $2012,91(2012)$

9. Pilyugin, S: Shadowing in Dynamical Systems. Lect. Notes in Math., vol. 1706. Springer, Berlin (1999)

10. Sakai, K, Sumi, N, Yamamoto, K: Diffeomorphisms satisfying the specification property. Proc. Am. Math. Soc. 138 315-321 (2009)

11. Hayashi, S: Diffeomorphisms in $\mathcal{F}^{1}(M)$ satisfy Axiom A. Ergod. Theory Dyn. Syst. 12, 233-253 (1992)

12. Dai, $X$ : Dominated splitting of differentiable dynamics with $C^{1}$-topological weak-star property. J. Math. Soc. Jpn. 64, 1249-1295 (2012)

13. Lee, M, Lee, S: Robustly transitive sets with generic diffeomorphisms. Commun. Korean Math. Soc. 28, 581-587 (2013)

14. Arbieto, A: Periodic orbits and expansiveness. Math. Z. 269, 801-807 (2011)

15. Yang, D, Gan, S: Expansive homoclinic classes. Nonlinearity 22, 729-733 (2009)

16. Abdenur, F, Bonatti, C, Crovisier, C: Non-uniform hyperbolicity for $C^{1}$-generic diffeomorphisms. Isr. J. Math. 183, 1-60 (2011)

17. Mañé, R: A proof of the $C^{1}$ stability conjecture. Publ. Math. Inst. Hautes Études Sci. 66, 161-210 (1987)

doi:10.1186/1687-1847-2014-170

Cite this article as: Lee: The ergodic shadowing property from the robust and generic view point. Advances in

Difference Equations 2014 2014:170.

\section{Submit your manuscript to a SpringerOpen ${ }^{\ominus}$ journal and benefit from:}

- Convenient online submission

- Rigorous peer review

Immediate publication on acceptance

- Open access: articles freely available online

- High visibility within the field

- Retaining the copyright to your article 